\title{
TOMOGRAFIA ELÉTRICA E MODELAGEM PSEUDO 3D APLICADA NA REAVALIAÇÃO DE UMA OCORRÊNCIA DE COBRE, BACIA DO CAMAQUÃ
}

\author{
Renata Augusta Sampaio Paes, mestranda em Geociências e Meio Ambiente, IGCE-Unesp \\ Cesar Augusto Moreira, Depto. de Geologia Aplicada - IGCE-Unesp \\ Matheus Felipe Stanfoca Casagrande, mestrando em Geociências e Meio Ambiente, IGCE-Unesp \\ Lenon Melo Ilha, Universidade Federal do Pampa
}

Copyright 2018, SBGf - Sociedade Brasileira de Geofísica

Este texto foi preparado para a apresentação no VIII Simpósio Brasileiro de Geofísica. Salinópolis, 18 a 20 de setembro de 2018. Seu conteúdo foi revisado pelo Comitê Técnico do VIII SimBGf, mas não necessariamente representa a opinião da SBGf ou de seus associados. É proibida a reprodução total ou parcial deste material para propósitos comerciais sem prévia autorização da SBGf.

\begin{abstract}
O presente trabalho discute os resultados obtidos com 0 uso combinado entre os métodos da Eletrorresistividade e Polarização Induzida, na reavaliação de uma ocorrência mineral de Cobre. Foram realizadas nove linhas paralelas de tomografia elétrica em arranjo Schlumberger, as quais permitiram a obtenção de modelos de inversão 2D, mapas e modelos pseudo-3D de isosuperfície. A análise combinada dos produtos gerados, permitiu o reconhecimento de áreas com alta cargabilidade e alta resistividade relacionadas a áreas de ocorrência de sulfetos disseminados e silicificação, corroborado pelas exposições em superfície, bem como a estimativa da morfologia do depósito. A delimitação de áreas promissoras a ocorrência de minério, permite a demarcação de alvos para detalhamento futuro, através de técnicas diretas, como sondagem e amostragem de rocha. Este procedimento é recomendado em atividade de exploração mineral, devido a rapidez e facilidade de operação com possibilidade real de redução de custos e aumento das chances de incorporação de novos depósitos.
\end{abstract}

\section{Introdução}

A exploração de recursos minerais é descrita como uma atividade de alto risco, com retorno a longo prazo, em que apenas uma parcela dos alvos prospectados evidentemente resulta em uma mina, um cenário em onde a maioria das acumulações minerais não apresentam valor econômico, assim classificada apenas como uma ocorrência mineral (Moon et al. 2006; Richards 2009). Porém a rentabilidade e o valor econômico de uma acumulação mineral são variáveis temporais, suscetíveis a demanda de mercado, logística, fatores geopolíticos entre outros, portanto a reavaliação de uma ocorrência mineral sob uma nova ótica do conhecimento e cenário socioeconômico distinto é um procedimento indicado e viável.

Tanto na prospecção de novos alvos, quanto na reavaliação de uma ocorrência já descrita, uma forma de reduzir custos e riscos e ampliar as chances de incorporação de um deposito mineral são as investigações por meio de ferramentas indiretas, como os métodos geofísicos. A partir do reconhecimento prévio dos indícios de mineralização, em associação com levantamentos geológico-estruturais e estudo da metalogênese mineral, os modelos pseudo-3D gerados com a aquisição de dados geofísicos, podem resultar além da estimativa da morfologia da acumulação mineral, sua extensão e lateralidade, no refinamento e direcionamento com maior precisão de alvos a serem detalhados de forma direta, sondagem e amostragem de rocha(Mussett and Khan 2000; Dentith and Mudge 2014; Lu et al. 2017; Zhang et al., 2017).

Diante do exposto, este trabalho visa a reavaliação da ocorrência de cobre Colônia Santa Bárbara, localizada na Bacia do Camaquã (Figura 1), prospectada incialmente pelo DNPM em 1964 (Bocchi, 1970), por meio do uso combinado dos métodos geofísicos da eletrorresistividade e polarização induzida.

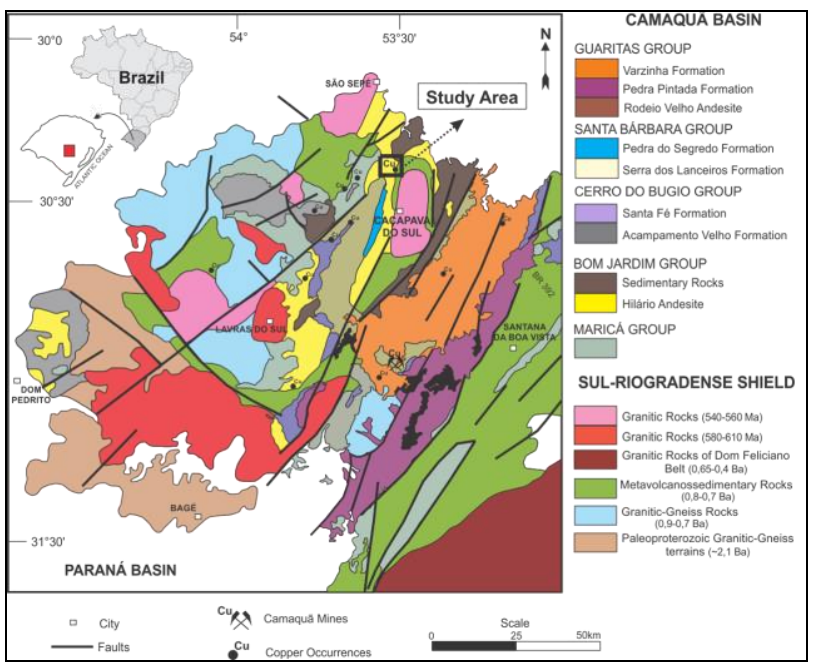

Figura 1 - Mapa Geológico simplificado da Bacia do Camaquã e do Escudo Sul-Riograndesnse, com destaque para a localização da ocorrência mineral em estudo (Modified from Paim et al. 2000 and Kazmierczak 2006).

Localizada em Caçapava do Sul - RS, a ocorrência foi reconhecida em trabalho de geologia de campo, devido a associação do cruzamento de duas falhas com orientação regional e ocorrência em superfície de barita, quartzo e calcita em associação de zonas brechadas com siderita e concentrações supérgenas de cobre, malaquita e calcosita. As análises geoquímicas realizadas apontaram para anomalia de cobre e as amostras dos dois furos de sondagem, alocados nas porções extremas do conjunto de falhas, registraram também cloritização e 
piritização em vários níveis, veios e fraturas, porém a área foi abandonada por apresentar níveis de cobre economicamente insatisfatórios.

A área de estudo é representada pela associação de rochas metassedimentares, do embasamento - Complexo Metamórfico Vacacaí, com andesito e tufo vulcânico do Grupo Bom jardim, unidade da Bacia do Camaquã (CPRM, 1995).

A bacia do Camaquã é historicamente conhecida pelo seu alto potencial poli-metálico, com a progressão do conhecimento decorrido da aquisição de novos dados e, vistas principalmente a solucionar os problemas encontrados na lavra do minério sulfetado das Minas do Camaquã, foram propostos inúmeros modelos metalogenéticos plausíveis para a origem dos depósitos da região. O presente estudo é desenvolvido com base no modelo de depósito magmático hidrotermal, proposto inicialmente por Carvalho (1932), Teixeira (1937) e Costa Filho (1944) com discussões retomadas por Remus et al. (2000) que confirma gênese ligada a uma fonte sedimentar com clara contribuição de uma fonte magmática.

\section{Metodologia/ Problema Investigado}

O planejamento geofísico foi realizado baseado na hipótese de uma mineralização disseminada no tufo vulcânico, da Formação Hilário, com metalogênese associada a ascensão de fluidos hidrotermais por meio de falhas e fraturas e facilitada pelo encontro das falhas existentes na área, com impregnação e estabelecimento das mineralizações nos poros da rocha hospedeira.

Inicialmente foi realizada etapa de campo para descrição de indícios de mineralização em superfície, seguida pela disposição de 9 linhas paralelas de tomografia de elétrica com direção N30E, afim de interceptar possível estrutura balizadora da ocorrência mineral e garantir maior cobertura e detalhamento da ocorrência (Figura 2).

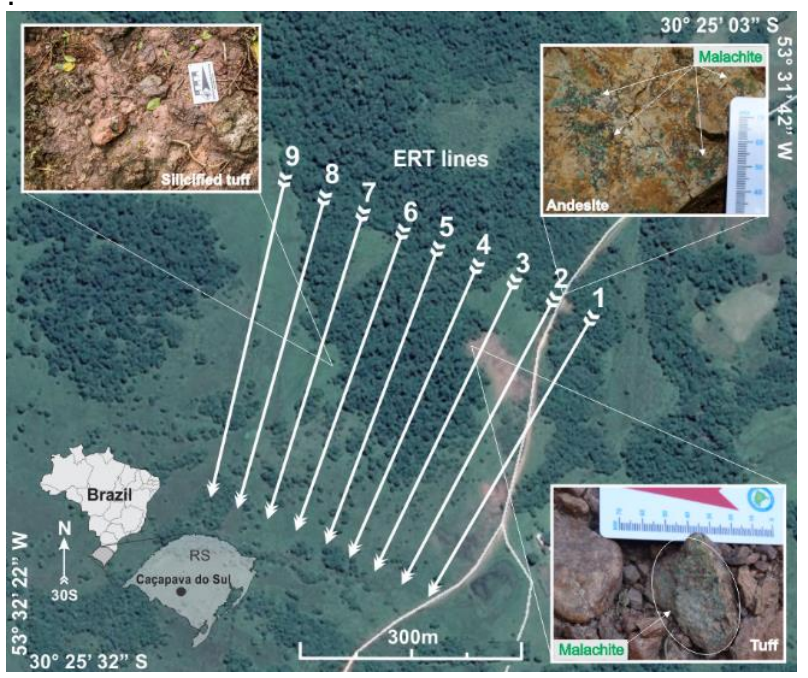

Figura 2 - Disposição das linhas de Tomografia Eletrica na área de estudo, com destaque para os indicios de mineralização descritos em superficie.
A aquisição geofísica foi realizada por meio do arranjo Schlumberger com separação de 10 metros entre eletrodos e extensão individual das linhas de 420 metros, para os métodos da eletrorresistividade e polarização induzida, por meio do equipamento Terrameter LS, fabricado pela ABEM Instrument, Suécia. Tal aparelho, conectado à uma bateria transmite e recebe sinais a partir de programação prévia, com $250 \mathrm{~W}$, resolução de $1 \mu \mathrm{V}$ e corrente máxima de 2,5 A (ABEM, 2012). Por meio de ciclos periódicos de transmissão de sinais (emissão de corrente de $500 \mathrm{~mA}$ por $1.5 \mathrm{~s}$, com duas janelas de medida de $100 \mathrm{~ms}$ cada e tempo de atraso de $0.5 \mathrm{~s}$ ), os ensaios são realizados e os dados registrados na forma de planilha (arquivo dat.), com possibilidade de ser formatada para tratamento futuro, acessada por interface USB.

Com os dados adquiridos foram gerados modelos de visualização 2D, por meio do programa Res2Dinv, pelo método inversão por suavização (smooth inversion), com correção topográfica. As seções de resistividade e cargabilidade são apresentadas em termo de distância $\mathrm{x}$ profundidade, com escala gráfica logarítmica de intervalos de valores com representação cromática.

Os produtos de inversão bidimensionais obtidos foram reunidos em uma única planilha e interpolados pelo método da mínima curvatura, e ajustados com a adição dos isovalores de superfície, através da através da plataforma Oasis Montaj, para geração de modelos de visualização pseudo-3D. Procedimento descrito em diversos estudos de uso combinado entre eletrorresistividade e polarização induzida (Moreira et al. 2012; Moreira et al. 2016; Vieira et al. 2016 and Côrtes et al. 2016).

\section{Resultados}

A análise conjugada dos dados de tomografia elterica obtidos durante o processamento dos modelos de visualização 2D, permitiram estabelecer uma única escala de valores para facilitar os estudos comparativos. Para cargabilidade foi definido uma escala entre 0.5 $\mathrm{mV} / \mathrm{V}$ e $3.54 \mathrm{mv} / \mathrm{V}$, e resistividade entre $1 \Omega \mathrm{m}$ e 1206 $\Omega . m$, intervalos que ressaltaram o contraste das zonas com relenvancia para o estudo, em que baixos valores são representados por cores frias e altos valores por cores quentes.

Afim de obter uma discussão mais detalhada, foram escolhidos como representativos da área em questao, por apresentarem claro os contrastes de propriedades fisicas existentes, os modelos das linhas 3, 4, 5 e 6 . As demais linhas são caracterizadas pela ausencia de contrastes evidentes e pela homodeinidade, utilizadas apenas para a interpolação e geração dos modelos pseudo-3D de visualização.

A seções de cargabilidade 3, 4 e 6 são marcadas por altos valores (acima de $\mathrm{mV} / \mathrm{V}$ ) na porção central, assim como a linha 5, que tambem apresenta altos valores na extremidade final do modelo. Com relação os modelos de resistividade, as seções apresentam altos valores (acima de $500 \Omega$.m) proximo ao centro, entre 160 e 220 metros ou 220 e 270 metros, as linhas 5 e 6 são marcadas 
também em suas extremidades finais por altos valores (Figura 3).

Afim de se obter uma visão mais integrada e espacial da ocorrência mineral, as linhas de inversão $2 \mathrm{D}$ de tomografia elétrica adquiridas em campo foram interpoladas e geraram modelos de visualização pseudo3D.

Foram obtidos mapas de resistividade e cargabilidade para as porções mais rasas e profundas, 140 e 50 metros de altitute respectivamente. O mapa de profundidade mais rasa apresentou predominante de uma zona central

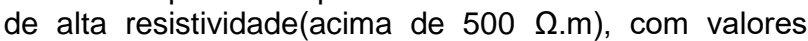
baixos circundantes, em adição de uma zona de alta cargabilidade (acima de $2 \mathrm{mV} / \mathrm{V}$ ) envolta de valores perifericos baixos. Os mapas de maior profundidade revelaram centro de alta resistividade comparativamente menor com os de baixa profundidade,entretanto, o núcleo de alta carbilidade aumenta em profundidade. Em ambos os casos é possivel identificar a atenuação radial dos valores para as porções mais externas (Figura 4).
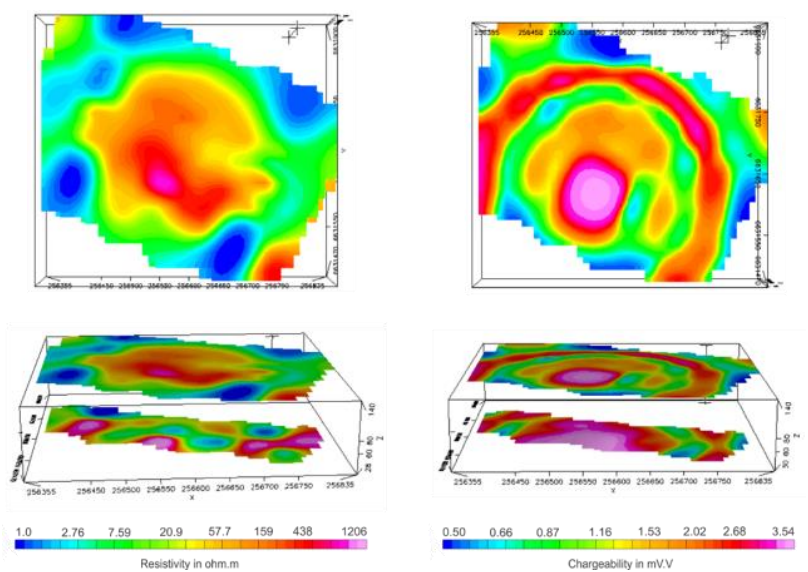

Figura 4 - Maps of resistivity (left) and chargeability (right) for the shallower and deeper positions of investigation.

\section{Discussão e Conclusões}

Silicificação é resultado da cristalização de sílica entre os poros da rocha, e pode ser, como neste caso, resultado da interação da rocha com fluidos quentes de origem hidrotermal. Porções silicificadas de rocha apresentam como propriedade alta resistividade, devido ao aumento da dificuldade ao trânsito de corrente nestas porções. 0 tufo vulcânico é uma rocha originalmente porosa, em que na área de estudo seus poros são preenchidos por sílica, assim como é o caso do metaconglomerado adjacente, intensamente silicificado.

Regiões com baixa resistividade são indicativos de bons condutores ou péssimos isolantes elétricos, o que em uma rocha porosa e contexto favorável podem ser indicativos de mineralização disseminada de sulfetos, acumulação de água ou veios e filoes de sulfetos.

Com relação a cargabilidade, zonas com sulfeto disseminado apresentam altos valores em relaçao a rocha estéril, logo regioes de baixa cargabilidade são indicativos de ausência de sulfetos dissemninados.

Baseado neste contraste de propriedades físicas que foram apresentados, as áreas mais promissoras para ocorrência de minério com valor econômico são as áreas caracterizadas por alta cargabilidade e baixa resistividade. Entretanto já nas linhas de aquisição $2 \mathrm{D}$. somente a linha 5 apresenta uma zona central de baixa resistividade (160 $\Omega . \mathrm{m})$, mas com predominância dos valores de cargabilidade intermediários (Figura 3)

Afim determinar as áreas mais promissoras para a acumulação de sulfetos disseminados, foram gerados modelos pseudo-3D de isosuperfície, para isso foi fixado os valores para resistividade em $800 \Omega$.m e cargabilidade em $2.5 \mathrm{mV} / \mathrm{V}$. As isosuperficies apresentam exatamente os valores fixados, com gradual aumento para as porções mais internas.

Os mapas de visualização 3D para resistividade revelaram a existência de corpos independentes, com destaque para um corpo vertical, bem como de corpos periféricos com feições pouco definidas (Figura 5).

Os modelos de cargabilidade apresentam maior complexidade de morfologia, com padrão dificil de ser estabelecido. A integração de várias areas centrais com alta cargabilidade resultam na modelagem de um corpo estruturalmente conico vertical com ampla base, gargalos na posição intermediaria e tendência de abertura para o topo. As porções periféricas de alta cargabilidade apresentam interconexão parcial, variavel ao longo da profundidade invertigada (Figura 5).

A integração dos modelos pseudo-3 de isosuperficie indicam que o corpo cônico de alta cargabilidade $(2,5$ $\mathrm{mV} / \mathrm{V}$ ) engloba o núcleo vertical de alta resistividade (800 $\Omega$.m), na parte mais interna da estrutura é possivel conter acumulação de sultetos disseminados no tufo vulvanico impregnado pelo cimento silicoso, fato corroborado pela exposição de afloramentos de tufo vulcanico silicificado em áreas coincidentes com a do limite superior da estrutura conica, enquanto a zona anular de alta carganilidade tem seu corresponde em superfície as impregnações de malaquita em tufo vulcanico . Esta zona anular é possivelmente sujeita a um sistema de falhas, responsáveis por contatos laterais desta estrutura alta representada pelo tufo vulcânico no centro, com andesitos ao norte e metaconglomerados ao sul. Baseado neste fato, a hipótese genética que justifica as zonas mineralizadas e seus padrões morfológicos deve considerar a estruturação do terreno (Figura 5).

O sistema de falhas lateral ao tufo vulcânico possivelmente serviu como conduto preferencial para ascensão do fluido hidrotermal enriquecido em silica e metais, que com o resfriamento proporcionou a cristalização de sulfetos e quartzo nas zonas livres de pressão, planos de fraturas e poros da rocha (Pirajno 1992), resultando em um complexa configuração morfolófica nas zonas de alta cargabilidade e alta resistividade em profundidade.

A porção central do cone de alta resistividade e cargabilidade, aparentemente ocorre de forma desconecta da acumulação mineral do sistema de falhas. Neste caso, provavelmente houve a instalação de um sistema de falhas com ponto central coincidente com o centro da estrutura cônica, o qual pode até ter sido causado pela pressão de ascensão dos fluidos hidrotermais ou tais fluidos simplesmente aproveitaram algum sistema de juntas anterior a fase de mineralização. 
A faixa de valores de resistividade e cargabilidadeque possibilitaram a modelagem morfológica do corpo mineralizado, é muito menor que em estudos semelhantes a outras ocorrências minerais na região, que apresentaram valores médios de resistividade de

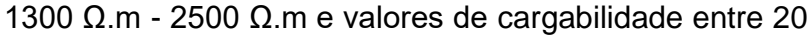
$\mathrm{mV} / \mathrm{V}-30 \mathrm{mV} / \mathrm{V}$ (Moreira et al., 2014, Moreira et al., 2016, Cortes et al., 2016 e Moreira e Ilha, 2011). Aparentemente, áreas com grande potencial econômico também apresentam baixos valores de resistividade, possivelmente por causa das maiores concentrações de minério (Moreira e Ilha, 2011 e Cortes et al 2016). Dessa forma, é provável que a ocorrência mineral em estudo tenha baixo potencial econômico, justificado por baixos valores de cargabilidade e ausência de baixas zonas de resistividade.

As principais conclusões deste trabalho permeiam a aplicação da rotina de pesquisa de detalhes proposta neste trabalho como uma alternativa viável e rápida nos procedimentos de exploração mineral de depósitos de sulfeto, com a possibilidade de ganhos reais em termos econômicos, e aumentar as chances de sucesso na descoberta de ocorrências minerais com valor econômico viável a extração.

Os resultados obtidos a partir de técnicas amplamente utilizadas na modelagem de depósitos na mineração, são satisfatórios para o detalhamento morfológico de uma ocorrência mineral de cobre. Produtos como mapas multiníveis, modelos pseudo-3D de isosuperfície permitem o detalhamento e a compreensão da morfologia mineral dos depósitos, além de uma previsão de seu potencial econômico, porém mesmo que baseado em evidências de mineralização em superfície, faz necessário a amostragem direta para análise quantitativa dos dados, como sondagem e amostragem de rocha.

A pesquisa de depósitos não-sulfetados com 0 procedimento descrito neste trabalho, precisa ser baseada em mais testes e estudos de viabilidade.

\section{Agradecimentos}

Os autores são gratos ao Conselho Nacional de Desenvolvimento Científico e Tecnológico (CNPQ), que através do Processo 470821/2013-2 (Edital Universal $\mathrm{CNPq}$ ) financiou este projeto.

\section{Bibliografia}

ABEM. (2012). Terrameter LS - Instruction Manual, ABEM Instrument, Sundbyberg, $122 \mathrm{pp}$.

Bocchi PR. (1970). Geological map of Caçapava do Sul, Rio Grande do Sul State, Bulletin DNPM 245, pp. 1-27 (in Portuguese).

Carvalho, PF. (1932). Reconhecimento geológico do Estado do Rio Grande do Sul. Boletim do Instituto Geológico e Mineralógico do Brasil, Rio de Janeiro, 66:1-72.

Cortes, A.; Moreira, CA.; Veloso, D.I.K.; Vieira, LB.; Bergonzoni, FA. (2016). Geoelectrical prospecting for a copper-sulfide mineralization in the Camaquã sedimentar basin, Southern Brazil. Geofísica Internacional, 55:165-174.
Costa Filho, JH. (1944). Prospecção das minas de cobre de Camaquã, Rio Grande do Sul. Boletim DNPM, n. 59: 13-94.

Dentith M and Mudge ST. (2014). Geophysics for the Mineral Exploration Geoscientist. Cambridge University Press, Cambridge, pp. 1-438.

Kazmierczak TS. (2006). Mapeamento da bacia do Camaquã com a utilização de dados geofísicos, geologia e sensoriamento remoto. MSc Dissertation Universidade Federal do Rio Grande do Sul.

Lu, DB., Wang, F., Chen, XD. et al. An Improved ERT Approach for the Investigation of Subsurface Structures, (2017). Pure Appl. Geophys. 174: 375. https://doi.org/10.1007/s00024-016-1386-9

Moon CJ, Whateley MEG and Evans AM. (2006). Introduction to Mineral Exploration. Backwell Publishing, Oxford, pp. 1-499.

Moreira CA and Ilha LM. (2011). Prospecção geofísica em ocorrência de cobre localizada na bacia sedimentar do Camaquã (RS). Revista Escola de Minas. Escola de Minas (64) 3: 305-311.

Moreira CA, Borges MR, Vieira GML, Malagutti Filho W and Montanheiro MAF. (2012). Geological and geophysical data integration for delimitation of mineralized areas in a supergene manganese deposits. Geofísica Internacional, 53: 201-212.

Moreira CA, Borssatto K, Ilha LM, Dos Santos SF and Rosa FTG. (2016). Geophysical modeling in gold deposit through DC Resistivity and Induced Polarization methods. REM International Engineering Journal, 69(3): 293-299.

Mussett AE and Khan MA. (2000). Looking into the Earth: an introduction to geological geophysics. Cambridge University Press, New York.

Paim P, Chemale JRF and Lopes RC. (2000). A Bacia do Camaquã. In: Geologia do Rio Grande do Sul. Rio Grande do Sul Federal University, Porto Alegre, pp. 231-274

Pirajno F. (1992). Hydrothermal mineral deposits: Principles and fundamental concepts for the exploration geologist. SpringerVerlag, Berlin, pp. 1-1250.

Remus MVD, Hartmann LA, Mcnaughton NJ, Groves DI and Fletcher IR. (2000). The link between hydrothermal epigenetic copper mineralization and the Caçapava Granite of the Brasiliano Cycle in southern Brazil. Journal of South American Earth Sciences, 13: 191-216.

Richards J. (2009). Mining, Society, and a Sustainable World. Springer, Heidelberg, pp. 1-506.

Teixeira, E. (1937). Cobre No Rio Grande Do Sul. Boletim Da Divisão De Fomento Da Produção Mineral, Dnpm, Rio De Janeiro, 22:29

Vieira LB, Moreira CA, Côrtes ARP and Luvizotto GL. (2016). Geophysical modeling of the manganese deposit for Induced Polarization method in Itapira (Brazil). Geofísica Internacional, 55: 107-117.

Zhang, G., Lü, QT., Zhang, GB., Lin PR. Jia ZY., Suo K. (2017). Joint Interpretation of Geological, Magnetic, AMT, and ERT Data for Mineral Exploration in the Northeast of Inner Mongolia, China. Pure Appl. Geophys. https://doi.org/10.1007/s00024-017-1733-5 


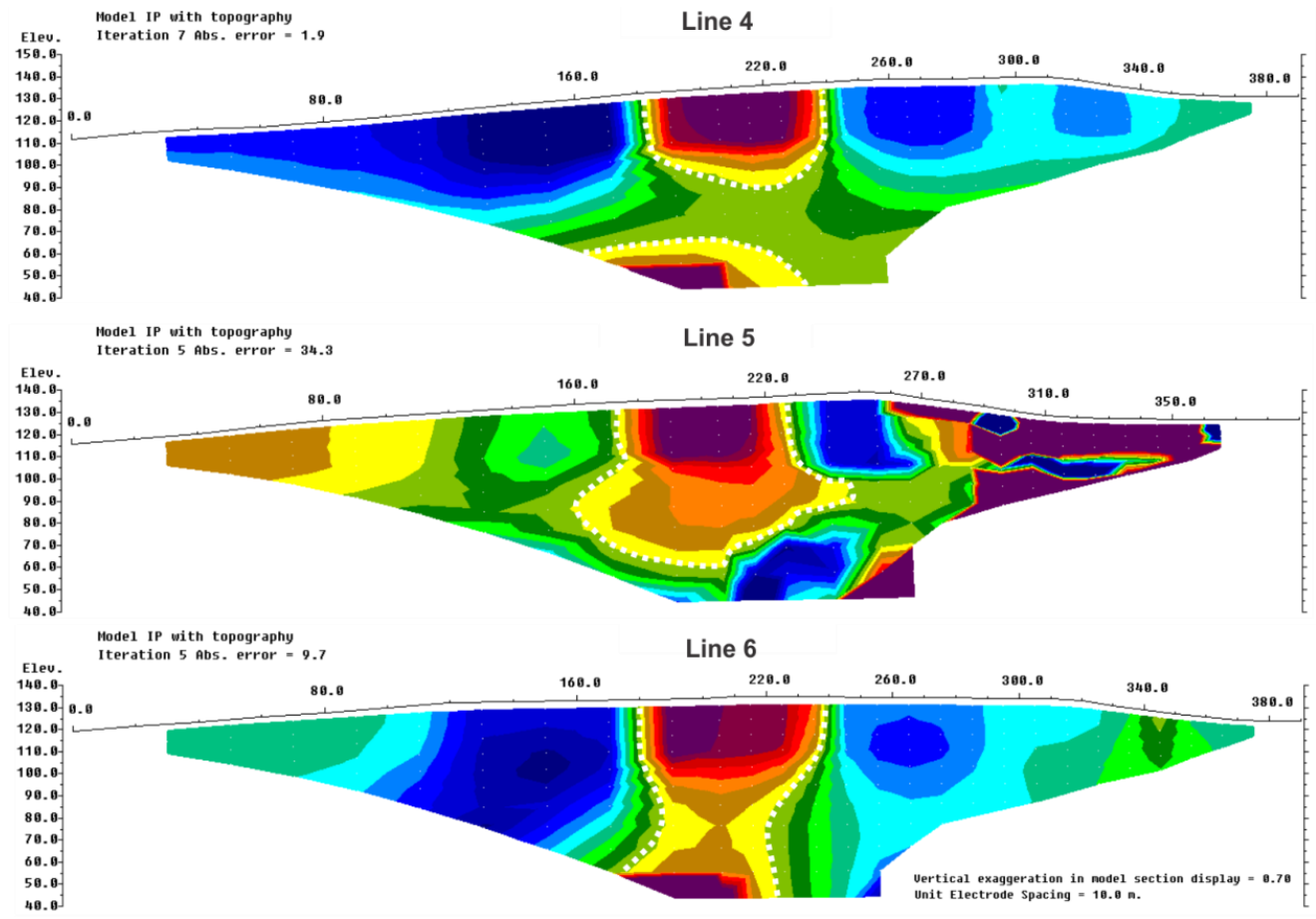

0.589 Charqeability in $\mathrm{nu} / \mathrm{U}$
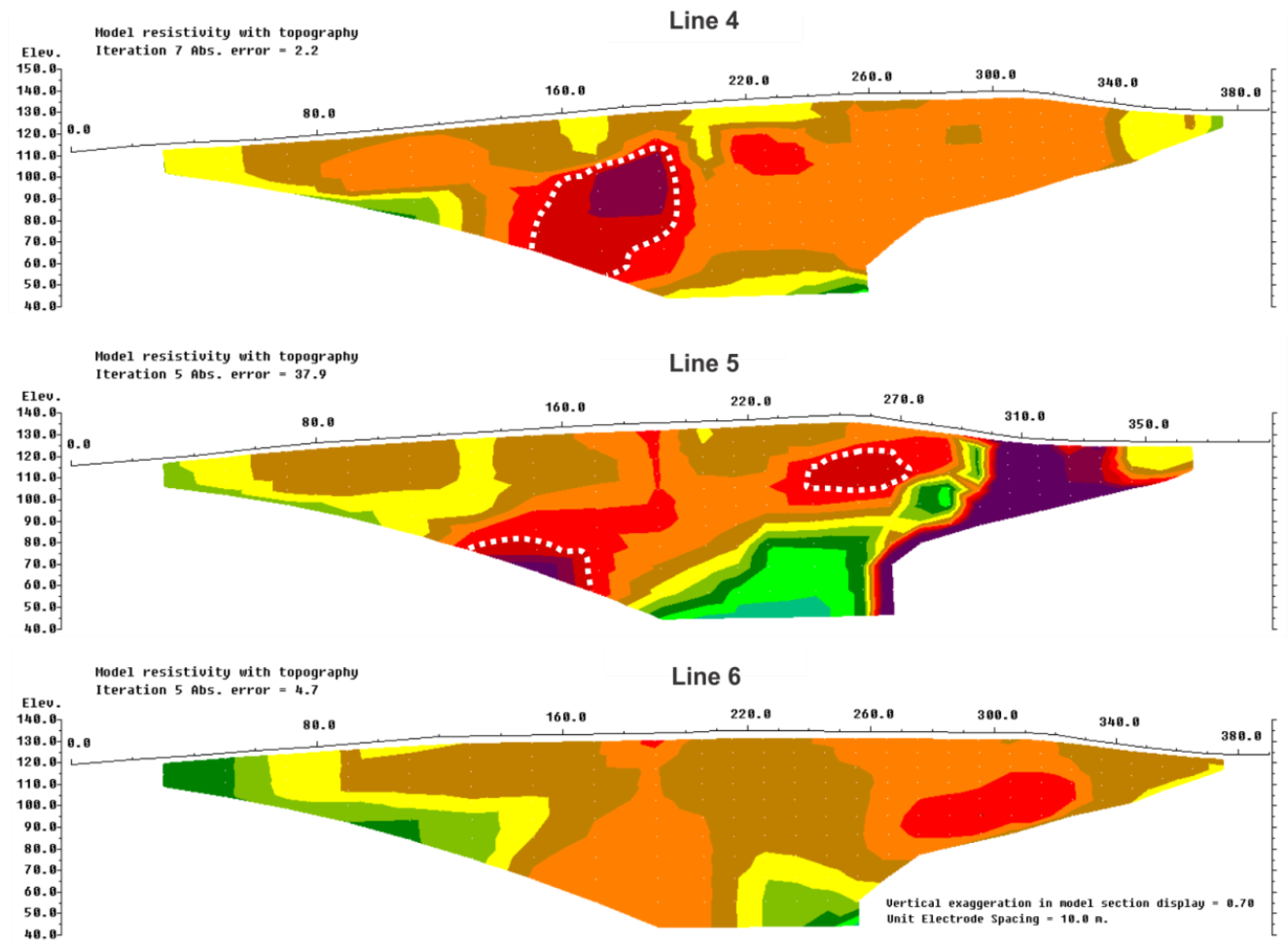

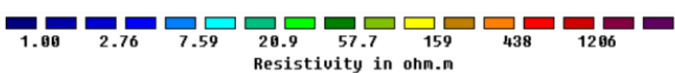

Figura 3 - Modelos de inversão de cargabilidade (acima) e resistividade (abaixo) para as seções representativas com maior contraste adquirido. 

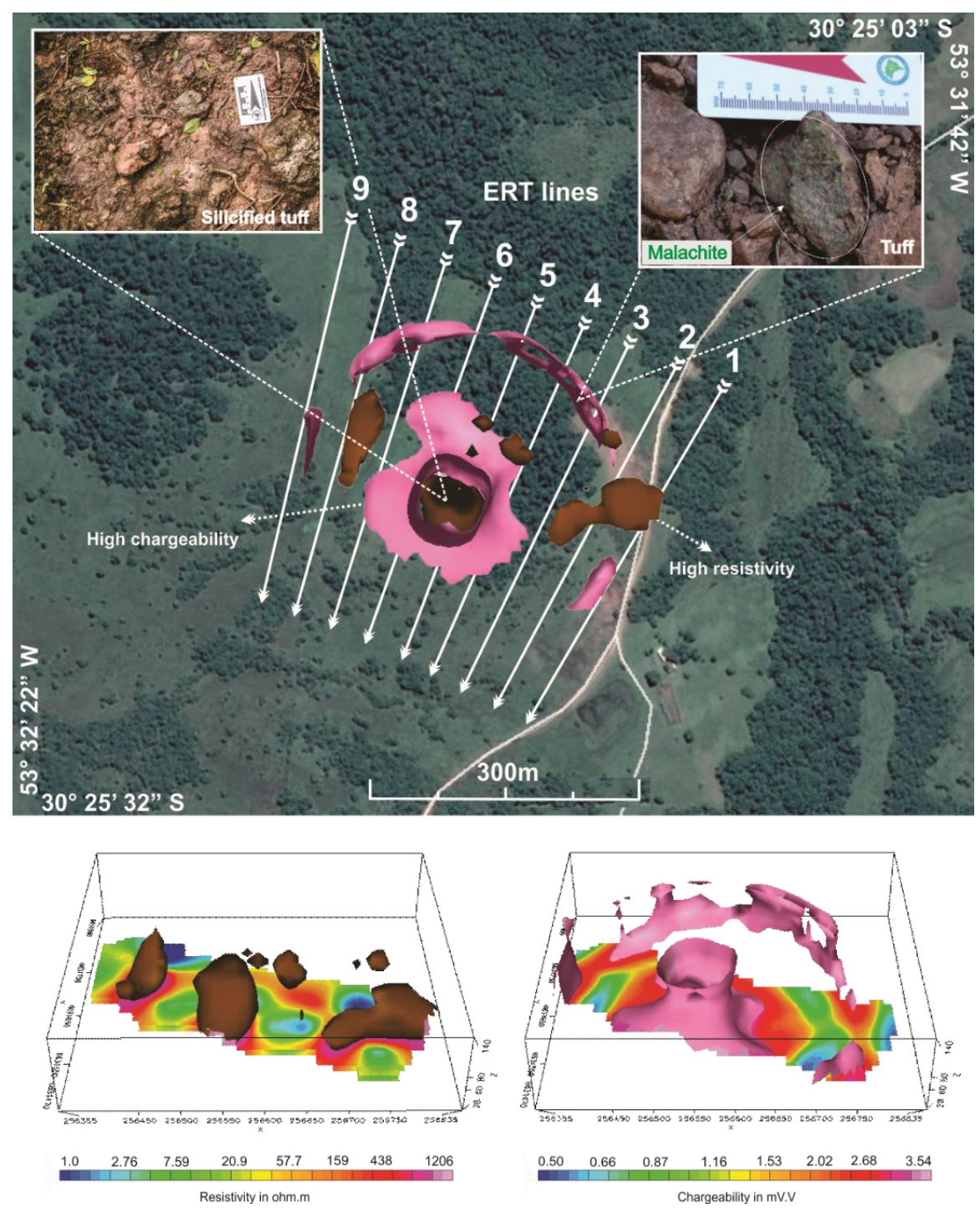

Figura 5 - Modelo pseudo-3D de isosuperfície, na direita para resistividade (800 $\Omega . m$ ) e esquerda para cargabilidade (2.5 mV/V), com integração dos modelos peseudo-3D de visualização para cada um deles. Ressaltando tufo silicificado representado pela zona de alta resistividade e impregnações de malaquita pela zona de alta cargabilidade. 